\title{
The "News Web Easy" news service as a resource for teaching and learning Japanese: An assessment of the comprehension difficulty of Japanese sentence-end expressions
}

\author{
Hideki Tanaka, Tadashi Kumano and Isao Goto \\ Science and Technology Research Labs. of NHK \\ 1-10-11, Kinuta, Setagaya, Tokyo, Japan \\ \{tanaka.h-ja, kumano.t-eq, goto.i-es\}@nhk.or.jp
}

\begin{abstract}
Japan's public broadcasting corporation, NHK, launched "News Web Easy" in April $2012{ }^{1}$. It provides users with five simplified news scripts (easy Japanese news) on a daily basis. This web service provides users with five daily simplified news scripts of "easy" Japanese news. Since its inception, this service has been favorably received both in Japan and overseas. Users particularly appreciate its value as a Japanese learning and teaching resource. In this paper, we discuss this service and its possible contribution to language education. We focus on difficulty levels of sentence-end expressions, compiled from the news, that create ambiguity and problems when rewriting news items. These are analyzed and compared within regular news and News Web Easy, and their difficulty is assessed based on Japanese learners' reading comprehension levels. Our results revealed that current rewriting of sentence-end expressions in News Web Easy is appropriate. We further identified features of these expressions that contribute to difficulty in comprehension.
\end{abstract}

\section{Introduction}

The convergence of TV and internet has enabled the creation of new services that allow users to overcome various temporal and spatial constraints (Hamada, 2013; Fu et al., 2006). It may even prove possible to effectively re-purpose content across different media. In this paper, we describe one such example: the application of TV news scripts for language teaching and learning on the internet.

\footnotetext{
${ }^{1}$ http://www3.nhk.or.jp/news/easy
}

Japan's public broadcaster, NHK, launched the News Web Easy web service in April 2012 (Tanaka et al., 2013). This site provides users with five daily simplified news scripts of easy Japanese news. Its purpose is to provide daily news to the foreign population in Japan, which has steadily increased to currently over two million. It would, of course, be preferable to provide news to these residents in their native languages. However, Japan's foreign population is so diverse that it would be virtually impossible to provide broadcasts in all of the expatriates' languages. NHK decided to tackle this issue by providing broadcasting services in simplified Japanese tailored to the language comprehension levels of foreign residents. Surveys among foreign residents have confirmed that a demand exists for broadcasts in easy Japanese (Yonekura, 2012).

News Web Easy's targeted audience in Japan comprises foreign residents learning Japanese as a second language ${ }^{2}$ who are already fairly fluent in conversational Japanese, but who want to learn to read news articles and newspapers. Their Japanese is thus at a pre-intermediate level.

The easy Japanese news comprises regular news that is jointly rewritten by a Japanese language instructor, with special training in easy Japanese, and a reporter. They closely adhere to the basic vocabulary and sentence patterns listed in the test guidelines of the Japanese-Language Proficiency Test (JLPT) (The Japan Foundation and Japan Educational Exchange and Services, 2002).

The test measures learners' Japanese proficiency at four levels ranging from level 4 (elementary) to level 1 (the most advanced) ${ }^{3}$. The vocabulary lists and sentence patterns in the test guidelines are graded, and the instructors can consult

\footnotetext{
${ }^{2}$ For the sake of brevity, in this paper we use the terms "foreigners" or "foreign residents" to signify foreign residents learning Japanese as a second language.

${ }^{3}$ The test has now been revised to cover five levels ranging from N5 (elementary) to N1 (the most advanced).
} 
these to verify the level of difficulty. News Web Easy employs vocabularies and sentence patterns ranked at levels 3 and 4.

NHK has received favorable comments about News Web Easy from foreign residents in Japan as well as from people overseas who appreciate this service for learning Japanese. Japanese language instructors also regard News Web Easy as a valuable educational resource. We believe that this favorable reaction is the result of the language level being set to an educational standard appropriate for Japanese. Moreover, the News Web Easy interface is similar to that adopted in language tutoring systems.

In this paper, we outline the features of News Web Easy and discuss its impact on language learning and education. We focus analytically on sentence patterns (sentences-end expressions) in news scripts that are not adequately covered in the JLPT test guidelines. Our analysis was based on an extensive collection of these materials compiled from a corpus of regular news texts and easy Japanese texts. We present an assessment of the difficulty levels of these expressions according to foreigners' reading comprehension levels ascertained from online questionnaires. Last, we consider the possibility of extending News Web Easy as a learning and teaching resource for native-level Japanese used within regular news scripts.

\section{News Web Easy and language teaching and learning}

In this section, we explain the features of News Web Easy and discuss its impacts on Japanese teaching and learning.

\subsection{Features of Japanese in News Web Easy}

\section{Target level}

We were involved in the production of News Web Easy content. Our aim was to ensure that while the news texts were easy to understand, they were as natural as possible. After many trials conducted by NHK the pre-intermediate level was found to be the lowest level necessary for achieving these aims. This level was consequently set by NHK as the target for Japanese rewriting. It approximately corresponds to the proficiency level ranked between levels 3 and 2 of the old JLPT, and between levels N3 and N2 of the new JLPT.

\section{Rewriters}

For the production of News Web Easy, five regular news texts are chosen daily and rewritten by a news reporter and a Japanese instructor who perform different tasks. While the reporter streamlines the news texts and retains only the core information, the instructor simplifies difficult expressions.

\section{Rewriting policies}

When rewriting news articles, reporters and Japanese instructors confine themselves as much as possible to elementary vocabulary and sentence patterns. As noted above, rewriters use the JLPT test guidelines to check words and sentence patterns. An editor is specially assigned for this purpose to News Web Easy.

It is noteworthy that reporters and instructors also use terms that are not listed. These include technical terms, proper nouns, and terms that frequently appear in news articles but are difficult to simplify.

\subsection{Features of News Web Easy interface}

In addition to the above-mentioned measures used to simplify Japanese, News Web Easy has several reading support functions, described below and shown in Figure 1.

\section{Furigana (ruby) characters}

Japanese text is a combination of Chinese characters (kanji), two types of Japanese phonetic symbols (hiragana and katakana), Latin characters (romaji), and numbers.

Kanji characters are notoriously difficult to master because there are so many of them and also because the same characters can be read in different ways depending on the context. Foreign residents often find themselves unable to understand the meaning of words written in kanji.

To assist them, very small hiragana characters, called furigana, are offered above all kanji characters in News Web Easy to indicate the pronunciation. This enhances the ability of foreign readers to understand the meanings of Japanese words, even if they are unable to read kanji.

\section{Glossaries}

The basic approach adopted by News Web Easy is to write simple Japanese using elementary vocabulary. However, it is not possible to simplify the vocabulary of all difficult terms. 


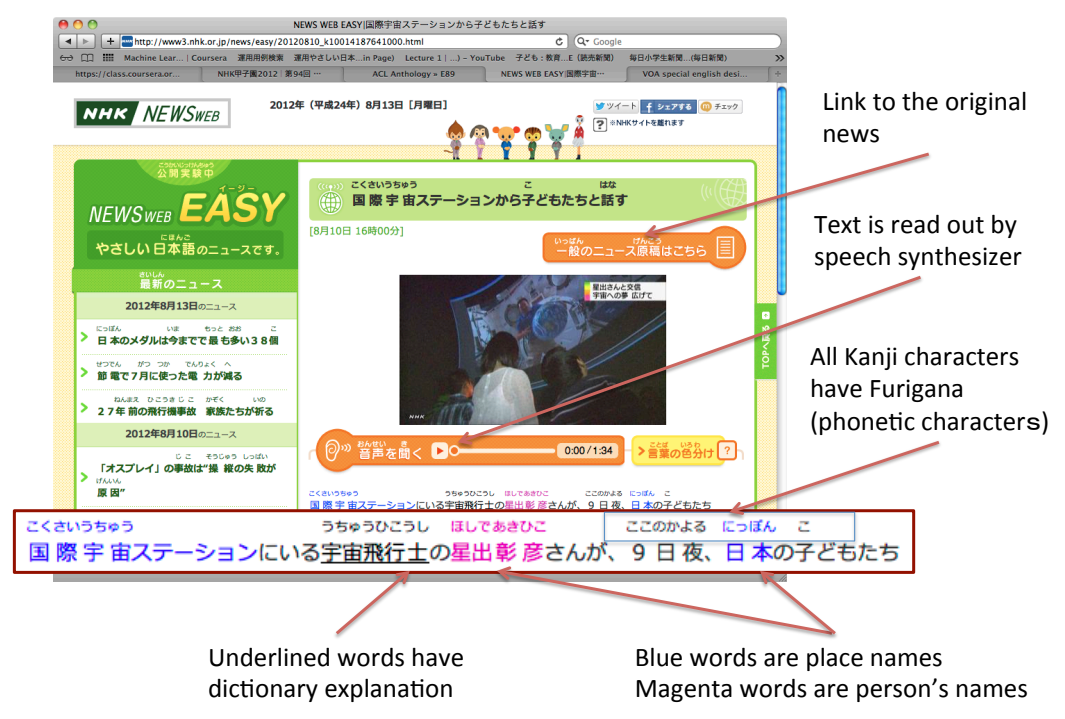

Figure 1: Screen shot of News Web Easy

News Web Easy resolves this issue by providing glossaries to explain difficult terminology. On the News Web Easy site, a glossary entry can be accessed by simply positioning the cursor over a word. A pop-up explaining the term is then displayed. A dictionary for Japanese elementary school students was used to provide the glossary entries.

\section{Proper nouns}

Proper nouns, not included within preexisting glossaries, inevitably appear in news articles. On the News Web Easy pages, different kinds of proper nouns are highlighted in different colors to capture the readers' attention. The reader may not know exactly what the terms mean, but at least this feature enables them to differentiate between the names of people, places, and organizations.

\section{Text to speech}

Some foreigners have difficulty reading Japanese, but are perfectly capable of understanding the text if it is read out to them. News Web Easy features a text-to-synthesized voice function to facilitate this mode of understanding.

\section{Links to original news}

Because News Web Easy reporters usually condense information from the original news item, full details are available through a link provided to the source web page.

\subsection{Teaching and learning}

The content produced by broadcasters is quite often used for language learning and teaching. Accordingly, it is important that News Web Easy contributes to this educational purpose as long as the main goal of providing news to foreign residents is not hampered. In this section, we discuss News Web Easy's contribution to Japanese teaching and learning.

\section{Contribution of Japanese texts}

News Web Easy essentially delivers "authentic" documents at a pre-intermediate level in natural Japanese. These texts are incorporated within automatic tutoring (learning) systems such as Reader-Specific Lexical Practice for Improved Reading Comprehension (REAP) (Brown and Eskenazi, 2004). Such documents attract keen interest among educators, although texts in languages other than English and French are rare (Uitdenbogerd, 2014). Thus, the simplified texts featured in News Web Easy are all the more valuable.

\section{Contribution of the interface}

The interface of News Web Easy offers reading support, as elaborated in section 2.2. Reading support is often used in language tutoring systems. For example, the Automatic Text Adaptation Tool (Burstein et al., 2007) automatically adds vocabulary support, automatic text reading by a speech synthesizer, summary text, and a transla- 
tion of the original text as "marginal notes." The reading support functions in News Web Easy can, therefore, be invaluable by providing simplified texts for pre-intermediate level Japanese learners.

The interface could also facilitate understanding of regular news for foreigners. As noted above, News Web Easy provides links to the original news stories so readers can compare both versions of the text. The scaffolding effect of providing simplified text for reading original text is widely recognized (Burstein et al., 2007; Eskenazi et al., 2013; Petersen and Ostendorf, 2007). Thus, this comparative reading should contribute to the comprehension of regular news.

\subsection{Language-level issues}

As noted in section 2.1, the News Web Easy rewriters currently use the JLPT test guidelines to check the language levels of words and sentence patterns in news items.

Because Japanese lessons typically start with the use of daily conversations, words and sentence patterns specific to news texts are often lacking in the JLPT test guidelines.

The rewriters have to judge for themselves the difficulty levels of words and phrases that are missing from the list. This could result in inconsistency in the language level of the simplified texts. Therefore, the content of the JLPT guidelines needs to be extended. As a first step toward this, we decided to focus on sentence patterns that were not included in the guidelines.

\section{Analysis of sentence-end expressions}

The sentence patterns in the JLPT test guidelines takes the form of a word sequence in the final positions of a sentence. We refer to this as sentenceend expressions. In the next section, we will define these and explain the features.

\subsection{Features of Japanese sentence-end expressions}

Japanese is a subject-object-verb (SOV) type of language in which predicates are positioned at the end position of a sentence. Japanese predicates usually contain one content word followed by some function words. Content words are typically verbs, nouns, and adjectives, and function words are auxiliary verbs, particles, formal nouns, and delexical (formal) verbs.
In this paper, we use the term sentence-end expressions (SEEs) to signify the function word sequence. SEEs add tense, polarity, voice, and modality to a sentence which we refer to as functional information, or simply as function. Such functions play an important role in deciding the meaning of a sentence.

SEEs may have more than one function lined up at the sentence end positions. We refer to such lined up functions as the function sequence (FS). An SEE, therefore, has a FS whose length is at least one ${ }^{4}$.

Because Japanese is an SOV type of language, SEEs may become quite long when the "O" is in an embedded sentence, as in $\mathrm{S}(\mathrm{SOV}) \mathrm{V}$. Let us consider a sentence with a single function of probability:

$\mathrm{X}$ 社は 来年の 利益を 3 倍にする かもしれない (probability).

Xsha wa rainen no rieki wo 3 bai ni suru kamosirenai (probability).

(X Inc. may (probability) triple their profit next year.)

This may be embedded in a sentence that ends with ということです (toiukotodesu) (people say), which has a hearsay function, as in:

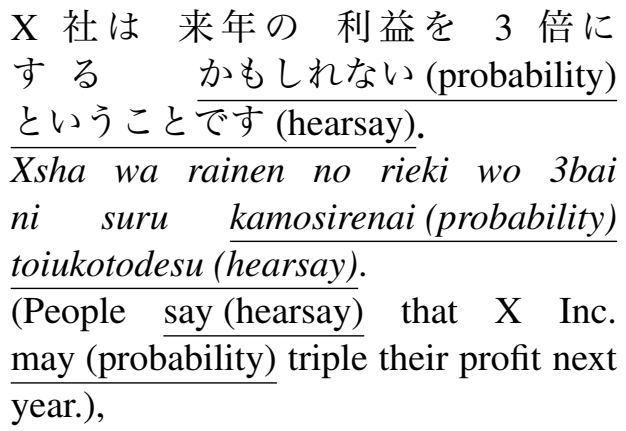

The English predicates in the above examples occupy different positions and do not have lined up functions. However, the Japanese predicates (SEEs) of both the main and subordinate clauses are linked to form a long SEE with the following FS: probability + hearsay (length 2). This complex structure is common in long Japanese SEEs and can be difficult for learners of the language to understand. We, therefore, consider SEE rewording to be essential for reducing the language difficulty level. We decided to extensively compile SEEs from regular news and News Web Easy to evaluate their difficulty for foreigners' comprehension.

\footnotetext{
${ }^{4} \mathrm{We}$ consider the number of functions in FS as the length.
} 


\begin{tabular}{lrrr}
\hline \hline & Regular & \multicolumn{1}{c}{ Easy } & Total \\
\hline Sentence Count & $3,937,214$ & 20,616 & - \\
SEEs & 477 & 775 & 1,063 \\
Meaningful SEEs & - & - & 841 \\
\hline
\end{tabular}

Table 1: Corpus size and SEE counts

\subsection{Compilation of SEEs}

We morphologically analyzed our corpus of regular news scripts covering a 16-year period and searched for SEEs. Our corpus contained about four million sentences. We only selected those that appeared over 100 times, resulting in a total of 466 SEE sentence types. Although our selection was restricted to the above frequency threshold, it still covered $98 \%$ of the total occurrence of all SEEs. Considering the corpus size, we found that SEE variation in the regular news was relatively limited.

We also extracted SEEs from our corpus of News Web Easy scripts, collected over a two-year period. This corpus contained about 20,000 sentences from which we obtained 755 SEE types. The total number of SEE types collected from both corpora was thus 1,063 . We then excluded SEEs with a plain statement, that is, SEEs that did not contain any meaningful functional information. This yielded 841 SEE types. Table 1 shows the corpus size and SEE counts.

\subsection{Functions specific to news scripts}

Before assigning a FS to each of the 841 SEEs, we first checked the SEEs and functions in a leading Japanese grammar textbook (Nihongo Kizyutu Bunpô Kenkyûkai, 2010). We found that some SEEs did not appear in that textbook and thus represented new functions that we termed objectivity and perception groups.

\section{Objectivity}

Two expressions-mono-da and koto-da-fell within this category. The formal noun, mono, has little meaning and simply refers to things in general. Another formal noun, koto, refers to general events. These terms are often added to simple factual statements in news stories, as in irei (exceptional)-no koto-da. Although it is possible to simply say irei-da, the addition of koto adds formality to the sentence. We believe this reflects the journalistic tendency of describing events as objectively as possible. We, therefore, termed this an objectivity function.

\section{Perception group}

Verbs such as mieru (seem), kiku (hear), and omou (think) entail the modality of how the speaker recognizes an statement's proposition. We thus referred to this modality as perception. We identified several SEEs that varied in objectivity and contained perceptions of third parties. Table 2 presents a list of SEEs with the perception group function.

The first expression, to-miteiru, comprises the content verb miru (see). The second expression, to-mirareteiru, is the passive version. Because, Japanese passive forms are often used without an agent (subject in a positive sentence), the person who does the seeing is not specified in this case. This lack of specification increases the level of abstraction of the sentence and adds objectivity.

The third expression, to-shiteiru, entails a delexical verb, suru (do) that ambiguously refers to miru (see), iu (say), and omou (think). This ambiguity further increases the level of abstraction and objectivity of the sentence.

The last expression, to-sareteiru, is the passivized version of to-shiteiru that we consider to have the highest level of ambiguity and objectivity.

Table 3 shows a list of all the functions used in this study. These are divided into functions of syntax, common modalities, and regular news specific modalities.

\subsection{FS assignment to SEEs}

To assign a FS to each of the 841 SEEs (described in section 3.2), we first compiled a set of regular expressions that linked function words to units bearing a single function. We then applied these regular expressions to the 841 SEEs and assigned a FS to each SEE.

Each SEE with a FS had a number of occurrence counts for each news type: regular and News Web Easy. We used these numbers to determine the association between FS and news type. An odds ratio was used to estimate the association:

$$
O=\frac{p}{1-p} \frac{1-q}{q}
$$

where $p$ is the relative frequency of a given FS in normal news and $q$ is the relative frequency of the FS in News Web Easy. A FS whose odds ratio was greater than or equal to 1 was considered to have 


\begin{tabular}{llll}
\hline \hline SEE & Function & Explanation & Objectivity \\
\hline to miteiru & percept. & see & low \\
to mirareteiru & pas.-percept. & be seen & middle \\
to shiteiru & amb.-percept. & do (see, say or think) & middle \\
to sareteiru & pas.-amb-percept. & be done (seen, said, or thought) & high \\
\hline
\end{tabular}

Table 2: SEEs bearing perception group function

\begin{tabular}{l|lllll}
\hline \hline Syntax & $\begin{array}{l}\text { causative } \\
\text { example }\end{array}$ & $\begin{array}{l}\text { passive } \\
\text { parallel }\end{array}$ & $\begin{array}{l}\text { aspect } \\
\text { nominalization }\end{array}$ & $\begin{array}{l}\text { give-get } \\
\text { noun }\end{array}$ & change \\
\hline Modality & hope & need & order & question & will \\
(Common) & selection & $\begin{array}{l}\text { prohibition } \\
\text { explanation }\end{array}$ & $\begin{array}{l}\text { invitation } \\
\text { change-guess }\end{array}$ & guess & probability \\
& reason & hearsay & & \\
\hline (News specific) & percept.group & objectivity & hearsay & & \\
\hline
\end{tabular}

Table 3: Functions assigned to SEEs

\begin{tabular}{lrrrr}
\hline \hline Length & \multicolumn{1}{c}{1} & 2 & 3 & \multicolumn{1}{c}{4} \\
\hline Regular & $9(0.14)$ & $35(0.54)$ & $21(0.32)$ & $0(0)$ \\
Easy & $13(0.16)$ & $43(0.51)$ & $22(0.27)$ & $3(0.04)$ \\
\hline
\end{tabular}

Table 4: The distribution of FS types

an association with normal news; otherwise it had an association with News Web Easy ${ }^{5}$.

Table 4 shows the number and relative frequency of FS types categorized by length and news type. The numbers for both news types peaked with the FS length of 2 and showed a similar distribution.

We calculated the relative frequency distribution of FSs using the same categories as in Table4. The results are shown in Figure 2.

Because FS may have occurrence counts in both news types, we calculated the average relative frequency for each one. We found that FSs assciated with News Web Easy had a high frequency concentration at length 1 , while FSs associated with regular news peaked at length 2 . We therefore concluded that SEEs with a single function were preferred in easy Japanese news.

Next, we compared the unique single functions that appeared specifically in each news type. We collected these functions from FSs of length 1 and the final functions in FSs of length 3 (see Table 4). Table5 summarizes these results. A sharp contrast is evident between the two types. Those functions

\footnotetext{
${ }^{5} \mathrm{We}$, therefore, considered the function sequence to be associated with regular news if $p$ was greater than or equal to $q$; otherwise, it was considered to be associated with News Web Easy.
}

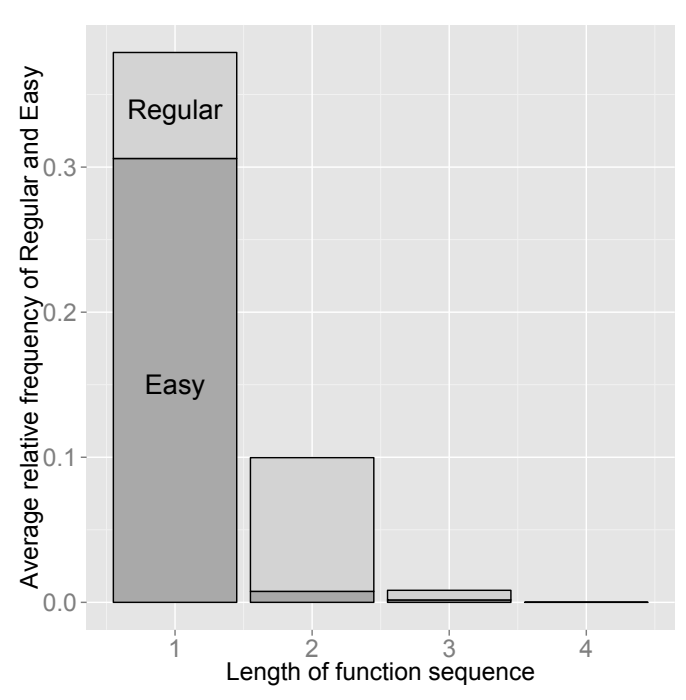

Figure 2: Average relative Frequency

used exclusively in regular news were all functions in the perception group. Those used exclusively in News Web Easy were syntactic types and modalities commonly used in daily conversation.

\section{Evaluation of SEE difficulty for foreigners}

\subsection{Measure of difficulty}

The difficulty levels of SEEs for foreign students were determined based on Japanese proficiency levels. This was measured according to the new JLPT version, using the lowest grade required to read and understand SEEs. Since the new JLPT has five grades, ranging from N1 (the most advanced) to N5 (elementary), we attached numbers 


\begin{tabular}{lll}
\hline \hline Length & Easy & Regular \\
\hline 1 & give-get & amb.-percept. \\
& order & pas.-percept. \\
& probability & pas.-amb.-percept. \\
& prohibition & \\
\hline 3 (final) & order & amb.-percept. \\
& explanation & pas.-percept. \\
& reason & \\
\hline
\end{tabular}

Table 5: Single functions unique to each news type

\begin{tabular}{cc|cc}
\hline \hline Grade & Number & Grade & Number \\
\hline N5 & 1 & N2 & 4 \\
N4 & 2 & N1 & 5 \\
N3 & 3 & above N1 & 6 \\
\hline
\end{tabular}

Table 6: JLPT levels and numbers for selection

ranging from 1 to 5 to them, with 5 indicating the most difficult SEEs and 1 the easiest. The number 6 was designated to SEEs that were difficult, even for N1-grade students. Table 6 presents the JLPT grades and numbers for the selection.

\subsection{Selection of SEEs}

We aimed to evaluate the difficulty levels of the 841 SEEs for foreign students learning Japanese and to analyze the factors governing these difficulty levels. The total number of SEEs (841) was too high to evaluate individually. Moreover, the word types for building SEEs were too diverse for the extraction of just a few factors.

We, therefore, decided to first sample FSs and then select SEEs bearing the sampled FSs. The number of FS types was 146 (Table 4) and that of function types was only 28 (Table 3 ) which would result in a highly tractable analysis of FSs. Accordingly, we selected SEEs based on the following assumptions and procedures.

(A1) Any FS belonging to the same cell in Table 4 would have the same difficulty level ${ }^{6}$.

Based on this assumption, we randomly sampled 13 FSs from the four cells in Table 4 of length 1 and 3, and for both news types. This resulted in a sample of $52 \mathrm{FSs}$.

(A2) Any SEE belonging to the same FS had the same difficulty level. Based on this assumption, we selected the most frequently occur-

\footnotetext{
${ }^{6}$ In other words, the difficulty level of FS only depends on the news type and the length of FS.
}

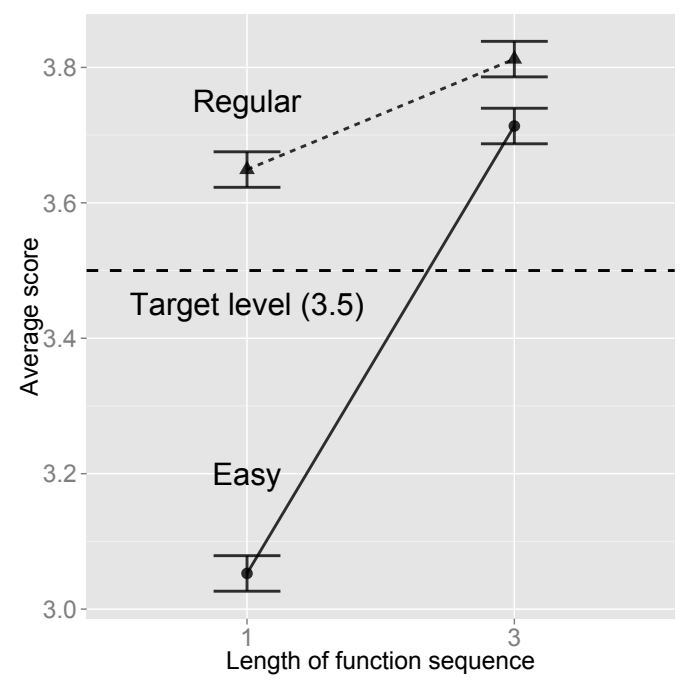

Figure 3: Score difference for the SEEs

ring SEEs from each of the $52 \mathrm{FSs}$ sampled in (A1) which also yielded 52 SEEs.

Because the regular news cell with a length of 1 had nine functions, we sampled 13 SEEs, allowing for FS duplication. It should be noted that the difficulty level of each sampled SEE and its FS was considered to be equal, because FS and SEE corresponded on a one-to-one basis.

\subsection{Subjects and questions}

We believed that foreign students-especially at the elementary level—would find it very difficult to respond to questions about their comprehension levels of SEE as they would need an in depth understanding of the functions to do so. We, therefore, asked Japanese instructors, and not the students, directly, to evaluate the difficulty levels of the SEEs.

Each questionnaire for the 52 SEEs consisted of the following parts: the SEE in question; functional features; and examples of usage in sentences. We sent questionnaires to 500 Japanese instructors through Internet. They specified the difficulty number in Table 6 for each of the 52 SEEs. In total, 390 effective responses were returned to us.

\subsection{Results and discussion}

Based on the responses of the 390 instructors, Figure 3 shows the average numbers (scores) calculated for the difficulty of SEEs and FSs obtained for the four cells (see Table4). The $\mathrm{x}$-axis shows the FS length and the $y$-axis shows the average 


\begin{tabular}{lll}
\hline \hline Function & News type & Score \\
\hline pas.-percept. & regular & 3.800 \\
pas.-amb.-percept. & regular & 3.791 \\
percept. & regular & 3.736 \\
noun & regular & 3.731 \\
amb.-percept. & regular & 3.701 \\
objectivity & regular & 3.513 \\
hearsay & regular & 3.485 \\
causative & regular & 3.369 \\
passive & regular & 3.354 \\
probability & easy & 3.346 \\
\hline
\end{tabular}

Table 7: The top 10 single functions according to difficulty levels

scores. The solid line indicates score changes for News Web Easy and the dotted line shows those for regular news. It should be noted that we set our target level of Japanese between N3 and N2 of the current JLPT. The target threshold score was 3.5.

The graph shows that the difficulty level of SEEs with a FS length of 1 from the News Web Easy cell was under the target threshold, while those in other cells were above this level.

\section{Effect of news type and FS length}

From that graph, it is evident that for both the lengths, SEEs obtained from News Web Easy were easier to comprehend than those obtained for regular news. We may conclude that the rewriting of the SEEs evidently reduced difficulty in understanding.

SEEs of a FS length of 3 were more difficult than those of a length of 1 for both types of news. As can be seen from the difference in the lines' gradients, the increase in difficulty associated with an increase in FS length was more apparent for SEEs from News Web Easy ${ }^{7}$. Although single functions used in News Web Easy evidenced low difficulty levels, these levels rose rapidly when they were lined up. Length of SEE is obviously one of the factors that affect the difficulty level.

\section{Effect of functions}

To confirm individual differences in FSs found in regular news and News Web Easy, we focused on the FSs with a length of 1 and arranged them, score-wise, in descending order (Table 7).

\footnotetext{
${ }^{7}$ The two-way analysis of variance test revealed that the difference was statistically significant.
}

It is evident from Table 7 that the nine single functions that appeared in regular news (see Table 4) occupied the top nine positions. In particular, the perception group functions were considered the most difficult. These were the ones that only appeared in regular news (c.f. Table 5). We can, therefore, conclude that many Japanese instructors consider the elimination of these functions in easy Japanese news to be an appropriate approach for maintaining the difficulty level below the pre-intermediate level.

\section{Conclusions and future work}

We were involved in NHK's web service initiative, News Web Easy. This initiative aims to deliver news in simplified Japanese to foreign residents learning Japanese as a second language. As we reported, the service has been welcomed as a Japanese teaching and learning resource. For this study, we analyzed features of News Web Easy that contribute to learning the language.

We focused on SEEs occurring in news most of which are not listed in JLPT test guidelines. We compiled an extensive collection of SEEs from regular news texts and News Web Easy and identified differences in SEE usage within regular news and News Web Easy. Consequently, we found a sharp contrast in terms of grammatical functions. We then examined the difficulty levels of these expressions for foreign students learning Japanese based on a wide-scale evaluation by Japanese instructors. Our results revealed that the current rewriting of SEEs is appropriate. Moreover, we identified features of these expressions that contribute to the difficulty factor.

A future challenge entails extending News Web Easy to make it a useful resource for those who wish to follow regular news that is written in native-level Japanese. Because News Web Easy facilitates comparative reading of both normal and easy Japanese, it offers such an opportunity to some extent. To further enhance this function, we believe that the findings of the present study will be valuable. The difficult SEEs that we found were appropriately reworded into simpler expressions and became unnoticeable in the simplified texts. If we can explicitly provide feedback about such information to News Web Easy users, they will be able to learn native-level Japanese more efficiently. Creating such an interface is, therefore, part of our future plans. 


\section{References}

Jonathan Brown and Maxine Eskenazi. 2004. Retrieval of authentic documents for reader-specific lexical practice. In Proceedings of InSTILL/ICALL Symposium.

Jill Burstein, Jane Shore, John Sabatini, Yong-Won Lee, and Matthew Ventura. 2007. The automated text adaptation tool. In Proceedings of Human Language Technologies: The Annual Conference of the North American Chapter of the Association for Computational Linguistics (NAACL-HLT), pages $3-$ 4, April.

Maxine Eskenazi, Yibin Lin, and Oscar Saz. 2013. Tools for non-native readers: the case for translation and simplification. In Proceedings of the Workshop on Natural Language Processing for Improving Textual Accessibility, pages 20-28, June.

Hsin Chia Fu, Yeong Y. Xu, and C.L. Tseng. 2006. Generation of multimedia TV news contents for WWW. In Proceedings of the 15th International Conference on World Wide Web, pages 909-910. ACM, May.

Hiroyuki Hamada. 2013. Overview of the hybridcast system. Broadcast Technology, 51:1-8.

Nihongo Kizyutu Bunpô Kenkyûkai, editor. 2010. Modern Japanese Syntax (Gendai Nihongo Bunpô) 1-7. Kuroshio Shuppan. (in Japanese).

Sarah E. Petersen and Mari Ostendorf. 2007. Text simplification for language learners: A corpus analysis. In Proceedings of ISCA SLaTE2007, pages 69-72.

Hideki Tanaka, Hideya Mino, Shinji Ochi, and Motoya Shibata. 2013. News services in simplified Japanese and its production support systems. In Proceedings of the International Broadcasting Convention 2013.

The Japan Foundation and Japan Educational Exchange and Services, editors. 2002. JapaneseLanguage Proficiency Test: Test Content Specifications (Revised Edition). Bonjinsha. (in Japanese).

Alexandra Uitdenbogerd. 2014. Tools for supporting language acquisition via extensive reading. In Workshop Proceedings of the 22nd International Conference on Computers in Education, pages 35-41.

Ritsu Yonekura. 2012. Information search and media access of foreign residents in Japan in disaster period-telephone interviews on four nationalities-. The NHK Monthly Report on Broadcast Research, pages 62-75, August. (in Japanese). 